\title{
Research and Analysis of Sports Training Real-Time Monitoring System Based on Mobile Artificial Intelligence Terminal
}

\author{
Biao Ma, ${ }^{1,2}$ Shangqi Nie, ${ }^{3}$ Minghui Ji, ${ }^{4}$ and Jeho Song $\mathbb{D}^{2}$ \\ ${ }^{1}$ Anhui Province Ying Shang County Shibalipu Town Center School, 236200 Fu Yang, China \\ ${ }^{2}$ Wonk Wang University, Sports Science, Iksan City, Republic of Korea \\ ${ }^{3}$ School of Physical Education, HuangHuai University, Zhumadian, China \\ ${ }^{4}$ Anhui Province Ying Shang County Balihe Town Center School, 236200 Fu Yang, China \\ Correspondence should be addressed to Jeho Song; sjhao@wku.ac.kr
}

Received 9 June 2020; Revised 10 August 2020; Accepted 27 August 2020; Published 28 September 2020

Academic Editor: Wei Wang

Copyright (C) 2020 Biao Ma et al. This is an open access article distributed under the Creative Commons Attribution License, which permits unrestricted use, distribution, and reproduction in any medium, provided the original work is properly cited.

\begin{abstract}
With the rapid development of artificial intelligence, related technologies and applications come into being, and industries based on artificial intelligence are booming, among which image recognition and target tracking technologies are widely used in various fields, especially in the fields of security monitoring and augmented reality. In this paper, combined with the characteristics of athletes, based on mobile artificial intelligence terminal technology, the $\mathrm{C} / \mathrm{S}$ mode of athlete training process monitoring system is developed and designed, which uses GPS to obtain the real-time position information of athletes and provide real-time guidance for athletes. In order to reveal the changing rules of various indexes of athletes in training state, the author makes synchronous tracking analysis from the aspects of individual sports function characteristics of athletes, training plan arrangement of coaches, brain function state, routine physiological and biochemical indexes, nutrition regulation, and injury conditions.
\end{abstract}

\section{Introduction}

It has long been a good and strong wish of mankind to thoroughly understand the mechanism of human intelligent behavior and to create intelligent machines that can simulate intelligent behavior [1]. During the long-term medical supervision with the sports team and the monitoring of athletes' physical function, a large number of original data have been generated, and the influence of various indexes on athletes' functional state is different in technology and algorithm [2]. Real-time monitoring of athletes' functional status and their positions in the training process and targeted determination of guidance programs and real-time guidance are very important means of scientific training today. A visual target tracking for mobile terminals is a research direction developed in the field of computer vision in recent decades [3]. The visual target tracking system of mobile terminal includes three main functional modules, namely, image acquisition module, image processing module, and image display module. The emergence and rapid development of artificial intel- ligence has brought hope to the realization of this wish [4]. Its research has prolonged the function of the human brain, deepened and expanded the intelligent labor of human beings, and made the scientific and technological revolution develop at an unprecedented speed. Many latest intelligent technologies, such as telepresence technology, virtual field technology, multi-intelligence technology, artificial neural network, multisensor fusion technology, and so on, have been applied in intelligent mobile robots.

Artificial intelligence is a frontier discipline, which is developed on the basis of computer science, cybernetics, information theory, systems science, philosophy, and other disciplines [5]. In recent years, mobile devices, especially mobile phones and tablet computers, have developed very rapidly. The chip processing speed of mobile devices is getting closer to personal computers. The memory capacity of mobile devices is almost the same as that of ordinary PC, and some even exceed PC $[6,7]$. If the athletes' functional information, position, road conditions, and other information can be mastered in real time during training, the best 
guidance scheme can be obtained through computer processing and provided to coaches, and the athletes can be communicated and guided in real time through wireless transmission; it will greatly change the current situation of middle-distance and long-distance running training in our country and improve the training quality [8]. Therefore, it is proposed to use computer technology and network technology as auxiliary means for data collection, data calculation, and conclusion analysis, and a real-time monitoring system for athletes' functional status is developed. The combination of sports and artificial intelligence is an inevitable trend of social development to conform to the new trend of the development of artificial intelligence in the world. This article hopes to attract more people to participate in the research of intelligent sports and provide new thinking for solving practical problems in sports.

The introduction part mainly introduces the popularity of mobile devices, the application status, and the wide application prospect of visual target tracking algorithm on mobile terminals. The related work in the second section summarizes the research status of visual target tracking in universities and scientific research institutions at home and abroad and briefly describes the key problems and solutions to be solved in the realization of mobile visual target tracking algorithm. The third part discusses the realization of a target tracking system based on the KCF-LD algorithm on the mobile artificial intelligence terminal, taking the KCF-LD algorithm as the target tracking module of the system, and introduces the design and implementation of the real-time monitoring system for physical training in detail. In the fourth part of this paper, the target tracking system implemented in this paper is used to experiment on real scenes, the hardware configuration of the experiment is listed, and the experimental scenes are classified. Finally, the experimental results are classified and compared, and some factors affecting the real-time performance of the system are analyzed, which proves the practicability of the KCF-LD algorithm in mobile terminals. At last, this paper summarizes the work done in this paper, expounds the innovation of this paper, and summarizes the shortcomings of the real-time monitoring system of sports training based on mobile artificial intelligence terminal, and looks forward to the future work.

\section{Related Work}

As computers have entered almost all fields more and more widely, it is an important response of computer intelligence to let computers exchange information with people in the way that people are accustomed to $[9,10]$. Although sports practical computer science has made rapid development in just over ten years and has effectively promoted the progress of sports, we have to calmly see that sports practical computer technology still lags far behind the development of computer science [11]. Anderson studied the related problems in the field of artificial intelligence [12]. Arkhipov proposes "Explanation of Artificial Intelligence: Insights from Social Science" research theory [13]. Santos proposed artificial intelligence: why we must do it well [14]. Let real objects be more vivid and easier to understand and at the same time enhance user experience. When augmented reality technology is applied to mobile terminals, real objects are displayed in the mobile devices in the form of images through cameras. Due to the movable characteristics of mobile terminals, objects of interest to users in the images will follow the moving positions $[15,16]$. However, for the time being, most coaches and researchers in our country focus their monitoring on athletes before or after training, and the monitoring during training only uses rhythm telemetry to monitor training. Limited by the data collection methods, the most basic artificial intelligence analysis can analyze the tracking of a single target and mine valuable information [17]. For example, in ball games, players control the trajectory of the ball to win the score. Therefore, how to design an effective human body detection algorithm to solve these two problems has become the focus of research in computer vision-related fields.

\section{Methodology}

The physical function of athletes is an important factor that determines their competitive level. It directly affects the performance of their athletic ability and technical level, thus affecting their athletic performance. The whole process monitoring system of athlete training developed and designed in this paper adopts $\mathrm{C} / \mathrm{S}$ architecture. The database of this architecture is composed of two parts: client application program and database server program, which are called foreground program and background program, respectively. The whole system consists of electromyogram collector, interface card, synchronizer and electromyogram acquisition, and analysis software [18]. For collective events, the tactical cooperation and tactical choice between athletes are important factors that determine the competition. It is necessary to continuously track and analyze the athletes' movements in the competition. The movable intelligent control terminal is used for intelligent control of household appliances, but most household appliances are currently realized by infrared communication. The infrared communication part of the system includes infrared emission and infrared receiving functions, and the infrared receiving function is mainly used for the learning function of the system on other remote controllers $[19,20]$. Human motion recovery of target video refers to using multiple cameras to capture human motion at the same time and recovering three-dimensional human posture through fusion processing of multiple data. Then, through computer programming, the computer simulates the reasoning thinking process (the process of selecting knowledge) peculiar to human beings, thus completing the intelligent problems that only human beings can solve.

Sixteen athletes were randomly and evenly divided into experimental group and control group according to male, female, and events. The basic conditions of the experimental group and control group were roughly the same. Before the experiment, the indexes of the experimental group and the control group were statistically tested. The results showed that there was no significant difference, and they were in accordance with statistics. Contrast requirements are shown in Table 1 and Figure 1. 
TABLE 1: Comparison of basic conditions between the experimental group and the control group.

\begin{tabular}{lcc}
\hline Group & Training years (years) & $5000 \mathrm{~m}$ score $(\mathrm{min})$ \\
\hline Experimental group & 5.3 & 16.25 \\
Control group & 6.4 & 15.33 \\
\hline
\end{tabular}

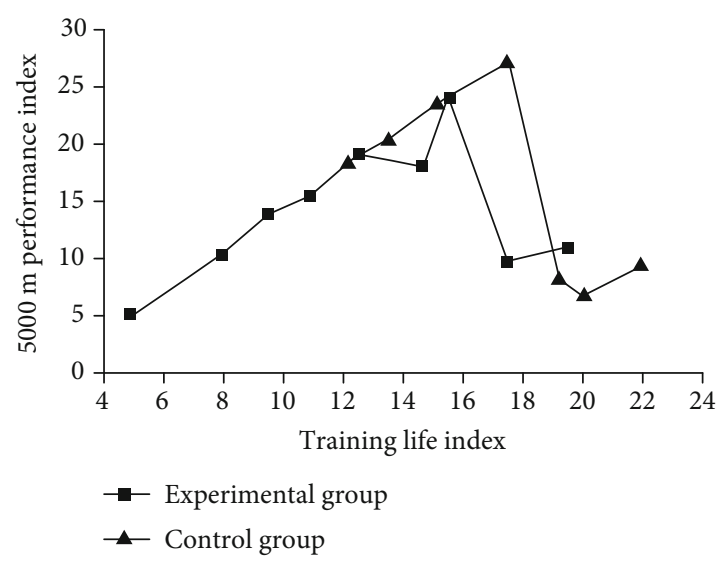

Figure 1: Comparison of basic conditions between the experimental group and the control group.

During the experiment, the GPS system was used to obtain the changes of athletes' basic sports quality in different positions in real time, and the wireless dialogue system was used to guide the athletes' training process and control their control intensity within the target range. This article takes the comparison results of $100 \mathrm{~m}$ and $50 \mathrm{~m}$ freestyle events as an example for analysis, as shown in Table 2 and Figure 2.

Spatial consistency means that the motion speeds of pixel points in adjacent positions are assumed to be consistent. This is a special assumption of optical flow method, because there is only one basic equation of optical flow method, but to find the velocity in horizontal and vertical directions according to the equation, only multiple equations can be solved simultaneously. According to the above assumptions, we can obtain the optical flow equation, see equation (1).

$$
F=K \times \frac{(V-V t)^{2}}{V} .
$$

Equation (1) indicates that the gray value of the pixel point remains unchanged after $V$ time, $K$, and $t$ displacement. The first order Taylor series expansion is carried out on the above equation to obtain formula (2).

$$
V=\sqrt{\frac{F \times V t}{K}+\frac{F^{2}}{4 \times K^{2}}}+V t+\frac{F}{2 \times K} .
$$

Assuming that the brightness is constant in a small local neighborhood of $I$, equation (3) is obtained.

$$
\beta_{x y}=\frac{I_{x y}}{\sum_{a-1}^{g-1} I_{x a}} .
$$

TABLE 2: Comparison of $100 \mathrm{~m}$ and $50 \mathrm{~m}$ freestyle performance between the experimental group and the control group after training.

\begin{tabular}{lcc}
\hline Group & Distance $(\mathrm{m})$ & Time $(\mathrm{s})$ \\
\hline Experimental group & 50 & 30 \\
Control group & 100 & 53 \\
\hline
\end{tabular}

Assuming $R_{i}$ is used to represent the motion speed of every pixel point, of course, the speed here is a vector that contains the magnitude and direction information of the motion speed of the pixel point; then, the above formula can be simplified as follows:

$$
R_{i}=M_{i} \sum_{i=1}^{N_{r}} \widehat{R}_{i, r}
$$

According to the present knowledge in the sports field, various artificial intelligence technologies and knowledge engineering methods are applied to deeply understand the internal semantics of user questions, mine the answers that users really care about, and correlate and recommend relevant knowledge that users may be interested in. Whether the imaging is fast and the system is smooth is the final decisive factor for the rationality of the architecture and algorithm design for the input reactants. With the continuous movement of the target in the scene, the target and the background near the target are constantly changing, so the template established for the target also needs to be constantly changed, which requires continuous learning of the target template in the tracking process and the establishment of a suitable model for the target. According to the overall design requirements, heart rate monitoring sensors, satellite positioning receivers, and language transmitters are developed and selected to realize real-time monitoring of various indexes of athletes' training process and obtain various information [21]. On the one hand, this evaluation can be used to judge the fatigue degree and recovery degree of athletes caused by sports training and to judge the fitness of athletes to sports load and the training effect during sports training. At the same time, we can know the changes of athletes' physical function, sports quality, and competitive ability at different positions at any time. We can use wireless cluster network to realize the communication between athletes and coaches during the training process. Athletes only need to wear a waterproof earphone to hear the guidance of coaches at any time.

After 3 months of experimental training, the 12 athletes in the experimental group and the control group were tested under the same conditions of time, place, and climate of their respective events. In order to avoid accidental factors, we conducted the same second test on the 10th day after the first test and compared the best results. The results show that the improvement of sports performance of the experimental group trained with real-time monitoring system is significantly better than that of the control group trained with traditional training (see Table 3 and Figure 3). 


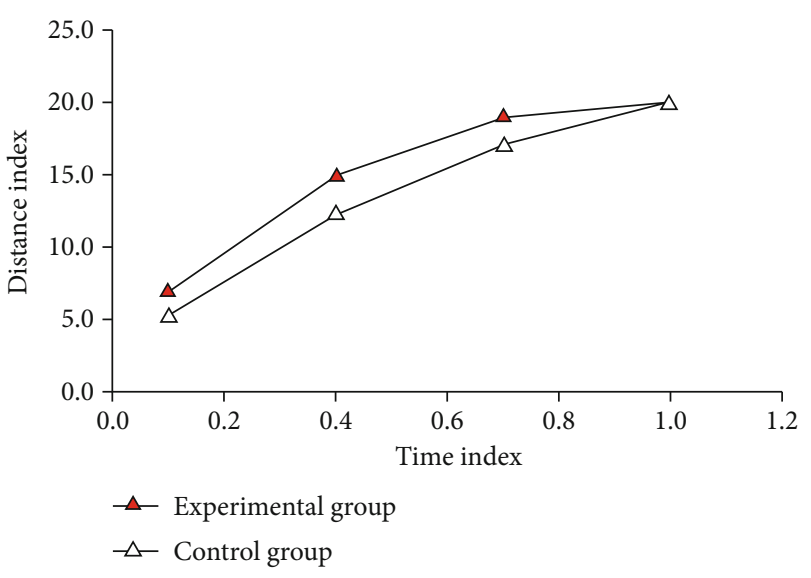

Figure 2: Comparison of $100 \mathrm{~m}$ and $50 \mathrm{~m}$ freestyle performance between the experimental group and the control group after training.

TABLE 3: Table comparing the growth of sports scores between the experimental group and the control group after training.

\begin{tabular}{lcc}
\hline Projects & Original score $(\mathrm{min})$ & Current score $(\mathrm{min})$ \\
\hline Marathon & 180.33 & 179.66 \\
$5000 \mathrm{~m}$ & 20.56 & 18.24 \\
Swimming & 2.03 & 1.65 \\
\hline
\end{tabular}

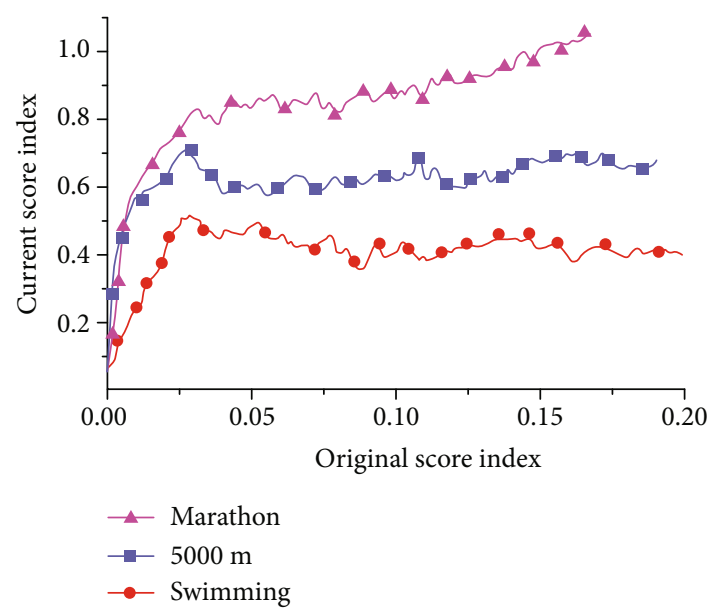

FIGURE 3: Comparative chart of sports performance growth between the experimental group and the control group after training.

Let the original target window be $D$, and its coordinates, width, and height are $D x, D y, D_{\text {width }}$, and Dheight. The candidate set takes $D$ as the original target and 1.3 as the scaling factor and makes 10 transformations with different scales. See formula (5) for enlargement transformation formula and formula (6) for reduction transformation formula.

$$
\begin{aligned}
& \left(D_{\text {width }}, D_{\text {height }}\right) * 1.2^{k} . \\
& \frac{\left(D_{\text {width }}, D_{\text {height }}\right)}{1.2^{k}} .
\end{aligned}
$$

In formulas (5) and (6), $k$ is $1,2,3,4,5,6,7,8,9$, and 10 , respectively, and the candidate window is guaranteed to be no less than 10 pixels in the scaling process.

Given a training sample set and labels $f_{i}$ and $X$, the training process of classifier $B$ is the process of minimizing the regularization loss function value by finding the best parameters. The linear classifier is generally expressed as equation (7).

$$
\begin{gathered}
B(\vec{X})=\prod_{i=1}^{m}\left(f_{i}(\vec{X})-f_{i}\left(\vec{X}_{w}\right)\right)^{1 / m} \\
R_{i}=M_{i} \sum_{i=1}^{N_{r}} \widehat{R}_{i, r}
\end{gathered}
$$

$m$ in the above formula represents the vector dot product, and minimizing the square error of the classifier for samples and labels is expressed as formula (8).

$$
B(\vec{X})=\prod_{i=1}^{m}\left(f_{i}(\vec{X})-f_{i}\left(\vec{X}_{w}\right)\right)^{w_{i}}
$$

A support vector machine and a regularization least square method are also based on this framework, in which SVM uses hinge loss function:

$$
B(\vec{X})=\prod_{j=1}^{n} u_{j}\left(x_{j}\right)^{v_{j}}
$$

Ridge regression in the least square method uses a square loss function:

$$
\omega_{s}=\frac{2 n \pi}{l} \sqrt{\frac{H}{m}}(n=1,2,3, \cdots) .
$$

As can be seen from the graphs in Table 4 and Figure 4, the KCF tracking algorithm lacks precision when dealing with target disappearance and low resolution, among which the TLD algorithm performs better when dealing with these two situations. If the target center error is allowed to exceed 35 pixels, TLD algorithm's precision exceeds KCF.

The interface card directly communicates with the computer and software through wired mode and sends commands and receives data to the electromyography collector and synchronizer in wireless mode at the same time. The collector is worn by athletes to collect electromyography, store data, and send in real time. In addition, the external contour loses image information that may be useful for understanding the target, such as texture and internal edge. For example, walking, trotting, and other actions have very similar human body contours on some image frames. The movable intelligent control terminal needs to accurately control the DC motor. A rotary encoder is introduced to detect the rotation feedback amount of the motor to judge the rotated angle, and the coordinates of the actual position of the motor are calculated through an algorithm and fed back to the control circuit. The synchronizer sends a measurement start and 
TABLE 4: KCF algorithm precision curve disadvantage table.

\begin{tabular}{lcc}
\hline Algorithm & Target center error & Accuracy \\
\hline KCF algorithm & 20.33 & 0.37 \\
TLD algorithm & 40.15 & 0.42 \\
\hline
\end{tabular}

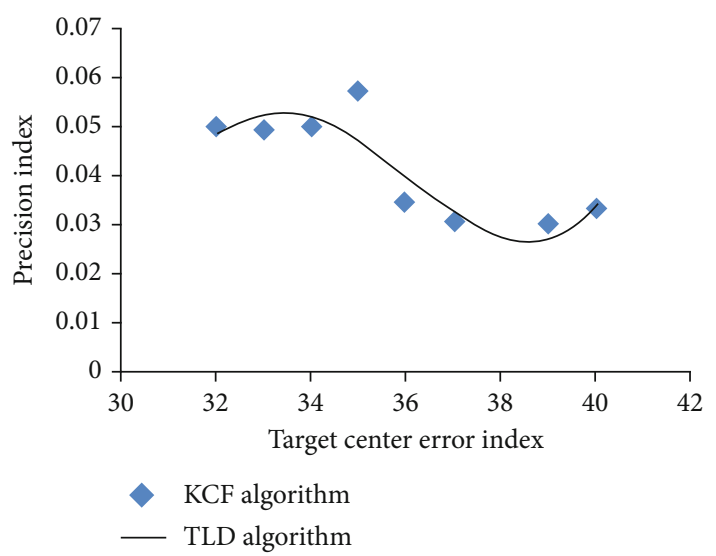

FIGURE 4: KCF algorithm precision curve inferior position chart.

end synchronization instruction to the camera or other equipment to coordinate the synchronization of each measurement equipment. The acquisition and analysis software performs measurement control, real-time monitoring, data management, and electromyography signal analysis. The technical and tactical analysis methods of different sports are different, but the basic steps are similar: first collect the original data, then extract the effective information, and then conduct in-depth analysis on the data. At the monitoring terminal, desktop geographic information system (GIS), mobile geographic information system, and virtual reality technology are used to present athlete's heart rate information and position information in the form of data. Meanwhile, spatial analysis technology is applied to analyze athlete's speed and heart rate at any position and time period. Through the use of computer network technology and artificial intelligence methods, real-time monitoring of athletes' functional states is realized, thus greatly improving the accuracy and efficiency of evaluation and diagnosis of athletes' functional states.

The following Table 5 and Figure 5 list the processing results of KCF algorithm tracking algorithm. From the figure, it can be concluded that KCF tracking algorithm performs better in dealing with deformation, occlusion, cluttered background, and other aspects; solves most of the key difficulties in target tracking; and has low computational complexity, which is suitable for application to mobile terminals.

The tracker of TLD algorithm uses optical flow method. KCF-LD replaces the tracking algorithm in TLD with the KCF tracking algorithm. The KCF-LD algorithm uses KCF tracker tracking result as reference and uses the nearest neighbor classifier to judge the tracking result. At this time, the detector is used to detect in the whole image. The method is still using the sliding window mode. All candidate windows obtained from the sliding window are input to the cascade classifier for screening. Finally, the remaining candidate win-
TABLE 5: Advantage table of precision curve of KCF algorithm.

\begin{tabular}{lcc}
\hline Processing contents & Target center error & Accuracy \\
\hline Deformation & 26.41 & 0.21 \\
Shelter & 30.12 & 0.35 \\
Cluttered background & 38.24 & 0.29 \\
\hline
\end{tabular}

dows are clustered to finally obtain the location of the target. The algorithm framework is shown in Figure 6.

Table 6 and KCF and DCF in Figure 7, respectively, show that the correlation filter with Gaussian kernel, and linear kernel is used for the kernel function. Table 6 shows that the correlation filter algorithm with Gaussian kernel function and directional gradient histogram feature has the highest accuracy, while the correlation filter algorithm with linear kernel function and directional gradient histogram feature has the fastest speed.

According to the characteristics of athletes' training center, rate information is obtained by heart rate monitoring sensors, real-time athletes' running distance, road conditions and positions obtained by differential global satellite positioning technology, etc. The tracker uses the median optical flow method to calculate the position of the target in the current frame according to the pixel states of the previous frame image and the current frame image. The detector scans the subwindows in the image. All subwindows pass through the cascade classifier and then filter out the background window. Because of the requirement of real-time performance, a simple noise suppression method is adopted in real-time detection, i.e., if the absolute value of the data is less than a predetermined threshold within a certain period of time, it is background noise, and the data is assigned a zero value. The sensor with the function of heart rate detection and sensing and GPS are integrated into a light and thin intelligent bracelet to be worn on athletes' hands. Through wireless cluster mobile communication technology, coaches only need to hold a microcomputer or a mobile PC to monitor various index data information in the training process of athletes. Highlight the individual characteristics of athletes, pay attention to the internal relations of various indicators of athletes, especially the dynamic changes of indicators according to the time sequence with the training process, from the characteristics of individual athletes' sports function. Its working principle is to use photoelectric conversion to replace the mechanical geometric displacement on the output shaft with the corresponding electric pulse or electronic digital quantity. The composition of photoelectric encoder includes grating disk, photoelectric detection, and other devices, wherein the grating disk is provided with fan-shaped light transmission channels uniformly on a circular plate with a certain diameter.

\section{Result Analysis and Discussion}

Single-feature human body descriptors based on machine learning are sensitive to noise interference, and multifeature fusion can further distinguish target objects that cannot be distinguished by single-feature description. Coaches' training 


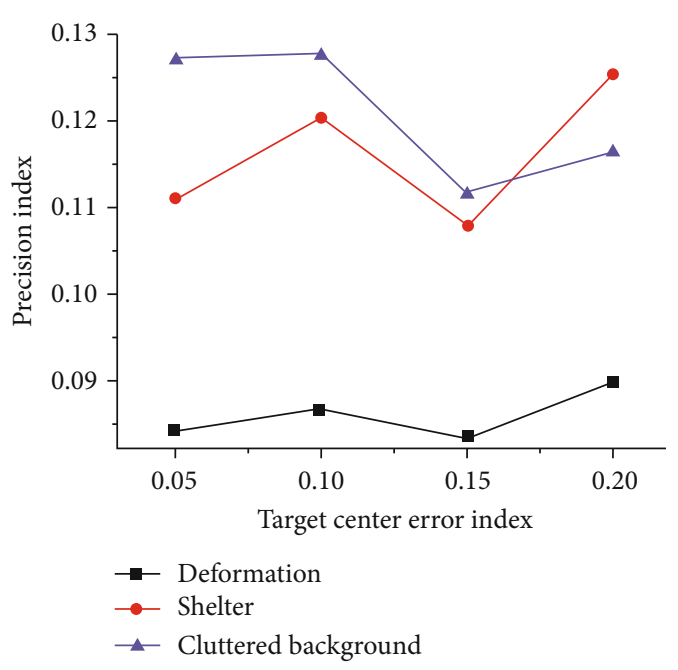

FIGURE 5: KCF algorithm precision curve dominance chart.

plan arrangement, brain function state, routine physiological and biochemical indexes, nutrition regulation, injury and illness conditions, and other aspects are systematically tracked and measured for a long time to find out the change rule of their function state. At the same time, it is possible to talk back to athletes through waterproof earphones equipped with voice transmitters in the athletes' ears, which will not affect athletes' training results. The noise caused by electromagnetic radiation is weakened by good shielding in hardware. A $50 \mathrm{~Hz}$ notch filter is used to filter out $50 \mathrm{~Hz}$ pollution, but the EMG signal frequency spectrum is concentrated in $20 \sim 200 \mathrm{~Hz}$, which filters out noise and EMG signal at the same time. By collecting the heart rate data of wheelchair racers throughout the season, the relationship between aerobic training, strength training, and athletic performance was studied. The concepts in the real world and the relationships between concepts are abstracted into entities and methods. The accumulation of knowledge instances is completed through entities, and the enrichment of knowledge expression is realized through methods. The construction of knowledge base can be completed more quickly based on customer historical data. Before training the weak classifier, set the same initial weight for each sample, generate a weak classifier for each round of training, and iteratively update the weight for each sample. Especially in the case of extremely high frequency or very steep pulse preface, more attention should be paid; only in this way can the influence of distributed capacitance between wires be reduced.

TLD, KCF, and KCF-LD are used to test the 26 target tracking reference videos. Two methods are used to determine whether an image is correctly tracked. The first method is based on the overlap degree. When the proportion of the overlap area between the tracking result and the real target position exceeds $25 \%$, the tracking is considered correct. See Table 7 and Figure 8 for the statistical results of tracking correct frames and accuracy based on overlap, and see Table 8 and Figure 9 for the statistical results of tracking correct frames and accuracy based on center offset distance.
High-reliability hardware foundation can ensure no hardware failure, but there are a large number of signal noise interference sources in life and production. The training samples are given initial weights, and important features with strong discrimination ability are selected from a large set of candidate features. The weights are updated in each iteration, and the weighted classification errors are used as the cost function to select features and add them to the overall strong classifier. Several target windows are obtained, and the learner integrates the results of the tracker and the detector and extracts the region most similar to the target from the integrated results to train the classifier so that the classifier can learn the latest appearance of the target. At this time, if the dynamic changes of athletes' heart rate are mastered through real-time monitoring and the heart rate information of the body responding to the exercise load is transmitted in real time through appropriate methods, the exercise intensity can be adjusted at any time to achieve ideal training effect. The choice of software system mainly considers its performance, function, price, practicability, compatibility, ease of management, etc. At the same time, it should also consider its future upgrade. Use single interface drive to enhance user experience. At the same time, the system can realize remote login. When coaches cannot supervise and guide athletes on the spot, they can monitor the whole training process and guide them in real time only through remote login.

Assuming that an $N \times 1$ one-dimensional vector is used to represent an image block, denoted as $x, x$ is taken as a reference sample, and the reference sample is shifted to obtain a series of negative samples, thus obtaining the positive and negative samples required for training the classifier. This operation can be represented by the following permutation matrix, see equation (11).

$$
H=\frac{1}{\sqrt{2}}\left[\begin{array}{cc}
1 & 1 \\
1 & -1
\end{array}\right] .
$$

The set can also be seen as consisting of two parts; half of the elements are obtained by forward cyclic shift of the reference sample, and the other half of the elements are obtained by reverse cyclic shift of the reference sample.

$$
H D=\frac{1}{l} \sum_{i=1}^{l} A_{i} \oplus B_{i} .
$$

If the vector $x$ is the pixel value of the first row in the image, a square matrix of $n \times n$ dimensions can be obtained by performing a cyclic shift operation on $x$; each row of the square matrix is a vector obtained by a cyclic shift operation of the vector $x$, and the square matrix can be expressed as formula (13).

$$
C \times\left(\sum_{i=1}^{I} q_{i, j}\right) \geq u_{j}
$$

The cyclic matrix structure is selected because when the classifier evaluates a plurality of different subwindows, the 


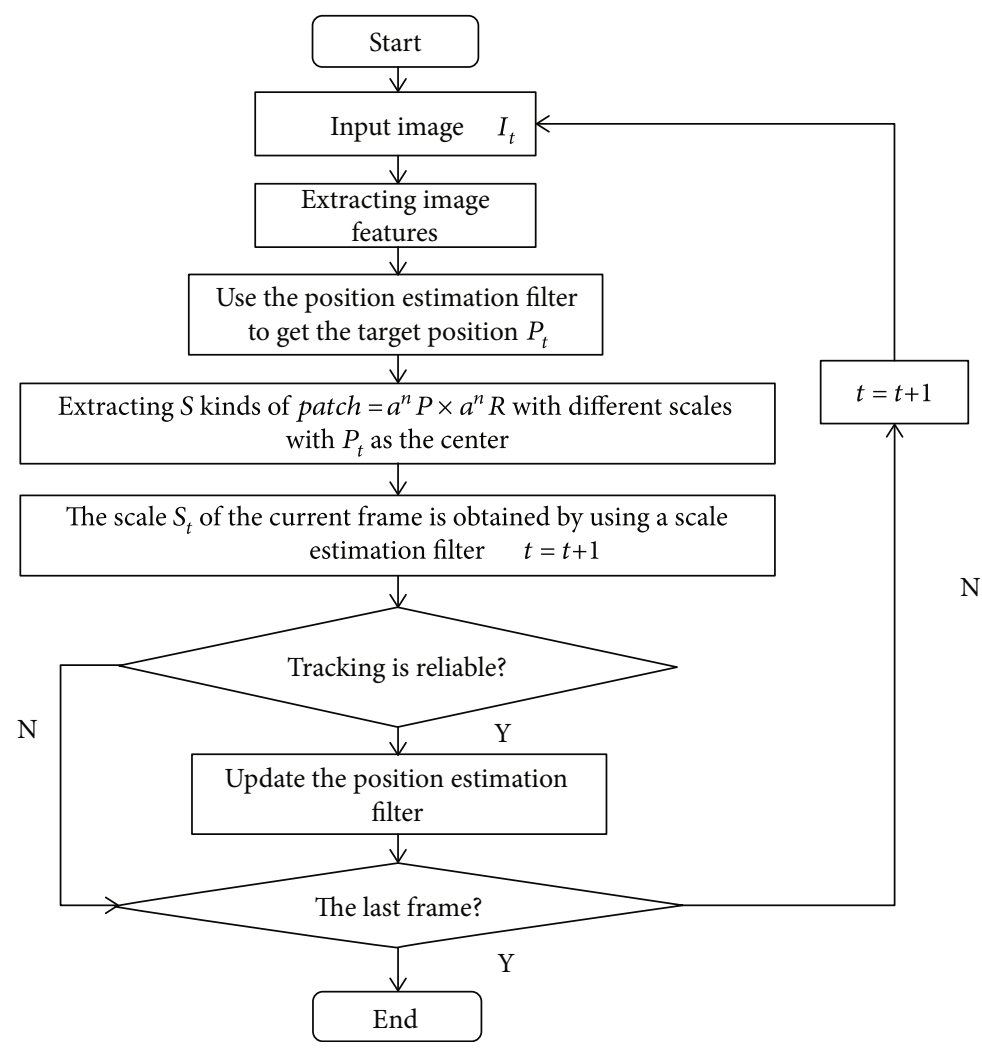

FIgURE 6: KCF-LD algorithm framework diagram.

TABLE 6: Comparison of average results between KCF algorithm and DCF algorithm.

\begin{tabular}{lcc}
\hline Algorithm & Average accuracy (\%) & Average frame rate \\
\hline KCF algorithm & 72.3 & 170 \\
DCF algorithm & 74.1 & 216 \\
\hline
\end{tabular}

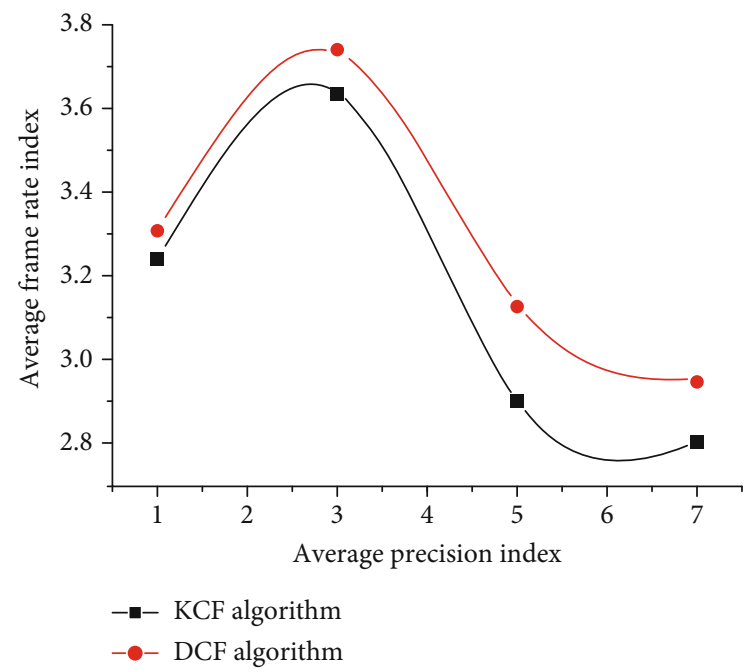

FIGURE 7: Comparison of average results between KCF algorithm and DCF algorithm. vector convolution of the cyclic matrix can be used to obtain the calculation result. If the product $C$ represents the convolution of the vector $u_{j}$ and the vector $q_{i, j}$, the result can be calculated in the Fourier domain:

$$
C \times\left(\sum_{i=1}^{I} q_{i, j} \times\left(\frac{f_{i, j}}{d_{i, j}+g}\right) 2\right) \geq u_{j} .
$$

This cyclic matrix structure can be converted into diagonal matrix by discrete Fourier transform, and this attribute is not related to the generation of reference sample $x$, so the cyclic matrix structure can be reexpressed as follows in combination with discrete Fourier transform:

$$
x(t)=A x(t)+B u(t) .
$$

If the above cyclic matrix structure is applied to linear regression, the training samples in equation (15) are all obtained by cyclic shift of the reference samples. It can be regarded as a noncentral covariance matrix, which is brought into equation (16) to obtain:

$$
y(t)=C x(t)
$$

In this experiment, the Volkswagen video where the target disappeared was first tested. In the experiment, the KCF tracker threshold value was set to 0.27 , and a smaller value was set because it has certain resistance to low resolution. On the other hand, it also indicates that the KCF-LD 
TABLE 7: Statistical results of correct frames and accuracy of tracking based on overlapping degree.

\begin{tabular}{lcc}
\hline Algorithm & Correct frame number & Accuracy \\
\hline TLD algorithm & 23 & 0.3 \\
KCF algorithm & 52 & 0.8 \\
KCF-LD algorithm & 29 & 0.4 \\
\hline
\end{tabular}

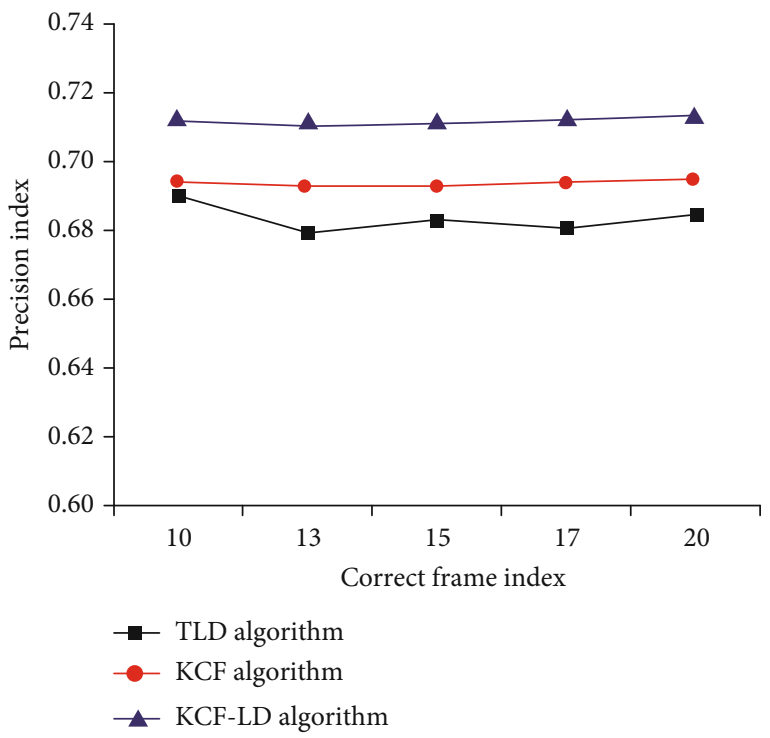

FIgURE 8: Statistical results of correct frames and accuracy of tracking based on overlapping degree.

TABLE 8: Statistical results of correct frames and accuracy of tracking based on center offset distance.

\begin{tabular}{lcc}
\hline Algorithm & Correct frame number & Accuracy \\
\hline TLD algorithm & 32 & 0.6 \\
KCF algorithm & 48 & 0.2 \\
KCF-LD algorithm & 26 & 0.5 \\
\hline
\end{tabular}

algorithm gives greater trust to the tracker in a low resolution video. Because the detector needs to pass through three-level classifiers when detecting several image blocks, the classifier with larger error will make the result of the detector inaccurate, thus affecting the tracking accuracy of the whole tracking algorithm. Figure 10 shows the test results of the target disappearance video Volkswagen.

Motion artifacts are mainly introduced by the relative sliding between the electrode and the skin and the motion of the connecting lead wire. The main frequency range of motion artifacts is concentrated in $0 \sim 20 \mathrm{~Hz}$, and the frequency spectrum of surface electromyography data is concentrated in $20 \sim 20 \sim 200 \mathrm{~Hz}$. Through the integration of indicators and comprehensive analysis, controlling the relationship between health status and fatigue degree in training not only helps to improve the training level but also forms the basis for evaluating whether players are suitable for playing and playing time. The weights of the training samples are

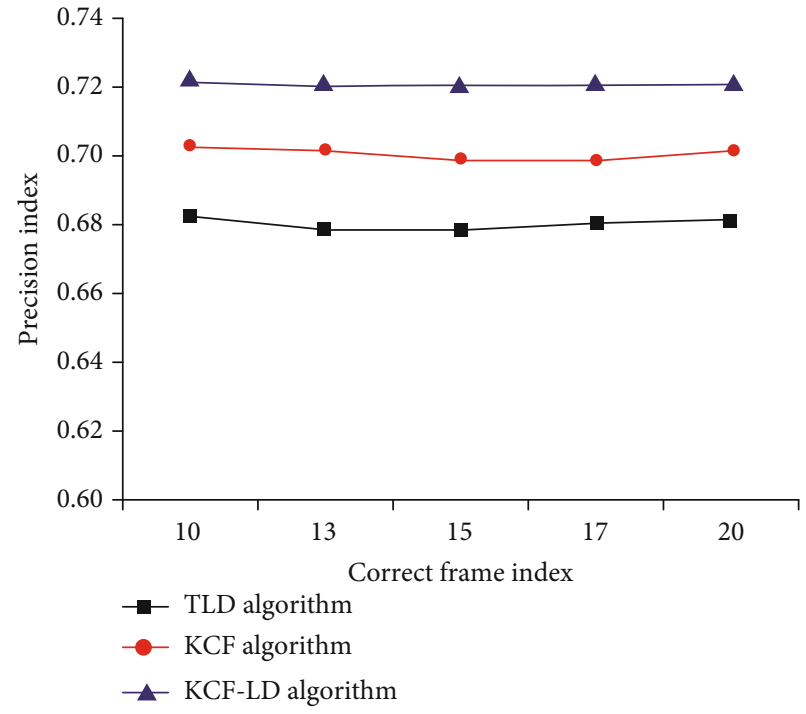

FIGURE 9: Statistical results of correct frames and accuracy of tracking based on center offset distance.

regarded as the same in each round, so all the features need to be trained once to form a weak classifier, and the classification results are calculated once and stored in the statistical table. Use shielded wire to connect the input terminal with weak signal, and the outer shielding layer of shielded wire must be well connected with common ground wire. When measuring with an oscilloscope, try to cut off the influence brought by the distributed capacitance of the connecting line. The signal connection mode is selected to be connected with the oscilloscope probe. Usually, the resting potential is deducted from the electromyography signal to eliminate the influence on the electromyography, but other factors are considered in this study; only the instrument bias is corrected, and the resting potential is not deducted. Although the team did not disclose the specific details, it is reasonable to believe that it is one of the means to help the team to make continuous progress by collecting physiological indexes through wearable equipment, continuously improving training parameters and optimizing training models through machine learning and using the results to guide the competition.

Using physiological and biochemical data indexes such as heart rate, blood lactic acid, blood urea, endocrine, and the like, the training process of athletes can be monitored in real time in a planned way, the adaptive characteristics of athletes can be mastered, and the scientificity of training implementation can be guaranteed. Due to the versatility of the development tool, it is very convenient to transplant the application program. When replacing the background database management system, only the appropriate database interface needs to be selected, and the application program itself does not need to be modified, which provides great convenience for future system upgrade and background database replacement. This is also the basis for monitoring training intensity by heart rate. According to this principle, we chose a mobile exercise heart rate monitor to carry out wireless heart rate monitoring to provide real-time heart rate during athletes' training for comprehensive integrated information. The task 


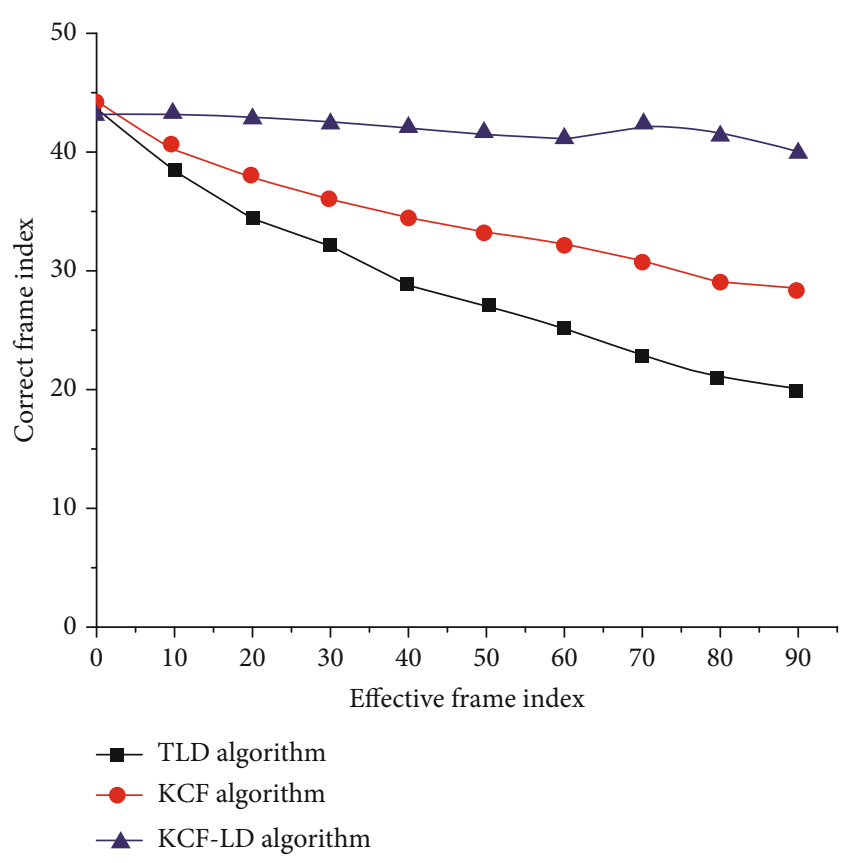

FIGURE 10: Tracking results of TLD, KCF, and KCF-LD on video Volkswagen.

of the tracking algorithm is to estimate the motion state of the target. The tracker first estimates the motion of some feature points in the rectangular region and estimates the reliability of these motions. The median of some of the most reliable motions is taken as the motion of the final rectangular frame. By testing whether the immune indexes are within the normal range, the body's health condition can be checked, and the possible health risks can be predicted. The change of EMG signal frequency can be regarded as fatigue analysis. Through the change trend of EMG signal frequency with time, whether the muscle is fatigue can be judged according to the physiological characteristics of the tested muscle. In this system, the athlete's position is directly displayed on the background of the electronic map. Taking the athlete as a geographical object, it can measure, analyze, and simulate the spatial information on which the athlete is based, thus indirectly obtaining the athlete's training status.

\section{Summary}

The establishment of training real-time monitoring system has realized the functions of obtaining athletes' heart rate information and position information in real time, processing and analyzing the information by computer, establishing a real-time scientific guidance scheme, and carrying out realtime information exchange. On the basis of fully considering the system structure, an intelligent mobile and learning system based on a single chip microcomputer is designed. Among them, the intelligent mobile function module integrates temperature and infrared sensors, as well as display and voice modules, which can automatically track and avoid obstacles and indicate the temperature, time, and date of the scene, making the design close to life. The establishment of real-time monitoring system for training combines the laws of human physiology, human movement, and modern science and technology, which has strong theoretical basis, practical application value, and scientific content. This system can monitor the training process of athletes online in real time and analyze and compare the training effect offline, which has guiding significance for the training process.

Because of the limitation of research time, methods, and experimental objects, it is necessary to make further research. At the same time, it is suggested that coaches of relevant departments and high-level sports teams can fully understand and participate in scientific training from thought to action so that the training level can reach a new level.

\section{Data Availability}

All the data used in this manuscript are included in the manuscript.

\section{Conflicts of Interest}

The author declares that there is no conflict of interest regarding the publication of this paper.

\section{Acknowledgments}

This paper was supported by Wonkwang University in 2020.

\section{References}

[1] Q. Guoyong, Z. Hucheng, and S. Yafei, "Management and application of high school students' physical fitness monitoring data," Physical Education, vol. 39, no. 6, pp. 60-61, 2019.

[2] Z. Hengliang, M. Wanli, and L. Hongyou, "Using data series to infer the analysis of the load data of college football special classroom teaching competitions," Sports Science Research, vol. 39, no. 6, pp. 83-90, 2018.

[3] C. Feng, H. Kun, and W. Lu, "Data analysis on physical health of primary school students in Wuhan based on monitoring of third-party organization," Hubei Sports Science and Technology, vol. 38, no. 1, pp. 4-7, 2019.

[4] L. Xiaolan, "Data collection system and recognition design of human motion," Computing Technology and Automation, vol. 38, no. 2, pp. 146-150, 2019.

[5] Q. Di and Z. Feng, "Research on the development of artificial intelligent sporting products," Sports Culture Guide, no. 6, pp. 104-108, 2018.

[6] L. Zhenhua, L. Yu, and W. Wenbo, "Research on the construction of early warning mechanism for college students' physical health," Liaoning Sports Science and Technology, vol. 40, no. 6, pp. 79-85, 2018.

[7] J. Wilkins, "Is artificial intelligence a help or hindrance?," Network Security, vol. 2018, no. 5, pp. 18-19, 2018.

[8] L. Xuan, "Application and research of LBS position trajectory tracking service platform in marathon movement," Science Teaching Journal, no. 8, pp. 249-250, 2018.

[9] T. Miller, "Explanation in artificial intelligence: insights from the social sciences," Artificial Intelligence, vol. 267, pp. 1-38, 2019.

[10] A. Curioni, "Artificial intelligence: why we must get it right," Informatik Spektrum, vol. 41, no. 1, pp. 7-14, 2018. 
[11] M. Milan, M. Branko, M. Dragan et al., "Artificial intelligence in clinical medicine and dentistry," Vojnosanitetski Pregled, vol. 74, no. 3, pp. 267-272, 2017.

[12] D. Anderson, "Artificial intelligence and applications in PM\&R," American Journal of Physical Medicine \& Rehabilitation, vol. 98, no. 11, pp. e128-e129, 2019.

[13] V. V. Arkhipov and V. B. Naumov, "Artificial intelligence and autonomous devices in legal context: on development of the first Russian law on robotics," SPIIRAS Proceedings, vol. 6, no. 55, pp. 46-62, 2017.

[14] O. C. Santos, "Artificial intelligence in psychomotor learning: modeling human motion from inertial sensor data," International Journal of Artificial Intelligence Tools, vol. 28, no. 4, article 1940006, 2019.

[15] G. Liang, X. Lan, J. Wang, J. Wang, and N. Zheng, “A limbbased graphical model for human pose estimation," IEEE Transactions on Systems, Man, and Cybernetics: Systems, vol. 48, no. 7, pp. 1080-1092, 2018.

[16] Y. Wang, "Design and development of sports video analysis system based on sports training," Automation Technology and Application, vol. 8, pp. 148-151, 2019.

[17] P. Shapshak, "Artificial intelligence and brain," Bioinformation, vol. 14, no. 1, pp. 38-41, 2018.

[18] P. Chemouil, P. Hui, W. Kellerer et al., "Special issue on artificial intelligence and machine learning for networking and communications," IEEE Journal on Selected Areas in Communications, vol. 37, no. 6, pp. 1185-1191, 2019.

[19] H. Feng and W. Dingjun, "Research on the solution of intelligent work order analysis system based on text similarity," Electronic Technology and Software Engineering, vol. 141, no. 19, pp. 222-223, 2018.

[20] L. Lu, "Frontier hotspot and evolution analysis of international sports artificial intelligence research based on Cite Space," Journal of Hebei Institute of Physical Education, vol. 2, pp. 37-44, 2020.

[21] Z. Bin, "Research on the design of sports training system under wireless sensor network," Computer Knowledge and Technology, vol. 14, no. 4, pp. 12-13, 2018, 21. 